\title{
Effects of ion concentration on the hydrogen bonded structure of water in the vicinity of ions in aqueous $\mathrm{NaCl}$ solutions
}

\author{
A NAG ${ }^{1}$, D CHAKRABORTY and A CHANDRA* \\ Department of Chemistry, Indian Institute of Technology, Kanpur 208016 \\ ${ }^{1}$ Present address: Department of Chemistry and Chemical Engineering, \\ California Institute of Technology, Pasadena, CA 91125, USA \\ e-mail: amalen@iitk.ac.in
}

\begin{abstract}
Molecular dynamics simulations of dilute and concentrated aqueous $\mathrm{NaCl}$ solutions are carried out to investigate the changes of the hydrogen bonded structures in the vicinity of ions for different ion concentrations. An analysis of the hydrogen bond population in the first and second solvation shells of the ions and in the bulk water is done. Although essentially no effect of ions on the hydrogen bonding is observed beyond the first solvation shell of the ions for the dilute solutions, for the concentrated solutions a noticeable change in the average number of water-water hydrogen bonds is observed in the second solvation shells of the ions and even beyond. However, the changes in the average number of hydrogen bonds are found to be relatively less when both water-water and ion-water hydrogen bonds are counted. Thus, the changes in the total number of hydrogen bonds per water are not very dramatic beyond the first solvation shell even for concentrated solutions.
\end{abstract}

Keywords. Aqueous ionic solutions; hydrogen bonds; solvation shells; molecular dynamics.

\section{Introduction}

It has been the common belief that the hydrogen bonding network of water can be perturbed significantly by the presence of ions and thus the hydrogen bonded structure of aqueous ionic solutions can be very different from that of pure water. A significant number of experimental studies such as electric field, calorimetric, conductivity, infrared and Raman spectroscopic measurements, extended X-ray absorption fine structural spectroscopy, neutron diffraction with isotopic substitution and nonlinear femtosecond spectroscopic methods have been employed over the years to elucidate the effects of ions on hydrogen bonded network in water ${ }^{1-13}$ In spite of all these experimental work, the issue of how ions affect the hydrogen bonded network of an aqueous medium beyond the first salvation shell remains an open question. Computer simulations perhaps stand as the most important tool to analyse the structural changes of aqueous ionic solutions for various ion concentrations because, in simulations, we can analyse the hydrogen bonded network in the different solvation shells separately, whereas most of the experimental techniques tend to explore an average behaviour.

*For correspondence
Although majority of these studies have supported the view of an altered hydrogen bonded structure in presence of ions and have put forward the notions of 'structure breakers' and 'structure makers', there are also some experiments which support the view of a very little structural change of hydrogen bonds beyond the first solvation shell of the ions. For example, recent experiments ${ }^{12,13}$ using femtosecond pump probe spectroscopy indicated that the rotational dynamics of water molecules are not affected by the ions beyond their first solvation shells even for concentrations as high as $6 M$ and, from this observation, it was inferred that there is very little effect of ions beyond the first solvation shell. A recent Raman spectroscopic study ${ }^{6}$ has also shown that the observed changes in the Raman spectra in presence of ions can be explained in terms of the strong electric field of the ions, the anions in particular, on the first solvation shell water molecules and no significant structural changes in the second solvation shell and beyond are reported. On the theoretical side, Guardia et $a l^{14}$ carried out a series of molecular dynamics simulations of infinitely dilute ionic solutions under ambient and supercritical conditions. Since the solutions were taken to be very dilute, expectedly no significant structural changes were found beyond the first solvation shell. Du et $a l^{15}$ looked at the struc- 
tural and dynamical aspects of concentrated alkali halide solutions. On the structural side, the focus was more on the changes of coordination numbers of cations and anions for different alkali halide solutions, and, to the best of our knowledge, a detailed simulation study of solvation shell-resolved changes of hydrogen bonds, taking both water-water and ion-water bonds into account, has not been made yet for highly concentrated ionic solutions. Such a study is presented here.

The organization of the rest of the paper is as follows. In $\$ 2$ we describe the simulation details. In $\S 3$, we discuss the equilibrium distribution of hydrogen bonds and the average number of hydrogen bonds in the first and second solvation shells of the ions as well as in the bulk water. Our conclusions are summarized in $\S 4$.

\section{Simulation details}

In the present work, the water molecules are characterized by $\mathrm{SPC} / \mathrm{E}^{16}$ potential and the ions are modelled as charged Lennard-Jones particles. ${ }^{17,18}$ In these models, the interaction between two atomic sites or ions are expressed as

$$
u\left(r_{i}, r_{j}\right)=4 \varepsilon_{i j}\left[\left(\frac{\sigma_{i j}}{r_{i j}}\right)^{12}-\left(\frac{\sigma_{i j}}{r_{i j}}\right)^{6}\right]+\frac{q_{i} q_{j}}{r_{i j}},
$$

where $q_{i}$ is the charge of the $i$ th atom (or ion). The Lennard-Jones parameters $\sigma_{i j}$ and $\varepsilon_{i j}$ are obtained by using the combination rules $\sigma_{i j}=\left(\sigma_{I}+\sigma_{j}\right) / 2$ and $\varepsilon_{i j}=\sqrt{\varepsilon_{i} \varepsilon_{j}}$ where $\sigma_{i}$ and $\varepsilon_{i}$ are the Lennard-Jones diameter and well-depth parameter for $i$ th atom or ion. The values of the potential parameters $q_{i}, \sigma_{i}$ and $\varepsilon_{i}$ for water and for the sodium and chloride ions are summarized in table 1.

In the starting configuration, the water molecules and ions were located on a face-centered cubic lattice with random orientations of water molecules. Each system, with a total of 500 molecules including ions at experimental density, ${ }_{19}^{19}$ was first equilibrated for $200 \mathrm{ps}$ in canonical ensemble at $298 \mathrm{~K}$ and subsequently the simulations were continued in the microcanonical ensemble for $400 \mathrm{ps}$ at an average temperature of $298 \mathrm{~K}$. The simulations were performed for three different concentrations of $\mathrm{NaCl}$. The long range electrostatic interactions were treated using the Ewald method with the dielectric constant of the surrounding medium $\varepsilon^{\prime}=\infty$ and the convergence pa- rameter $\alpha=6 \cdot 4 / \mathrm{L} .{ }^{20}$ The rotational equations of motion were solved using the quaternion formulation and for the integration over time, the leap-frog algorithm was employed with a time step of $10^{-15} \mathrm{~s}$ $(1 \mathrm{fs}){ }^{20}$

\section{Analysis of hydrogen bond statistics in different solvation shells}

The population analysis of the different shells around the ions required the definitions of the first and the

Table 1. Values of Lennard-Jones and electrostatic interaction potential parameters. $e$ represents the magnitude of electronic charge.

\begin{tabular}{llll}
\hline Atom/ion & $\sigma(\AA)$ & $\varepsilon(\mathrm{kJ} / \mathrm{mol})$ & Charge $(e)$ \\
\hline $\mathrm{O}$ & 3.166 & 0.6502 & -0.8476 \\
$\mathrm{H}$ & - & - & +0.4238 \\
$\mathrm{Cl}^{-}$ & 4.401 & 0.4184 & -1.0 \\
$\mathrm{Na}^{+}$ & 2.583 & 0.4184 & +1.0 \\
\hline
\end{tabular}

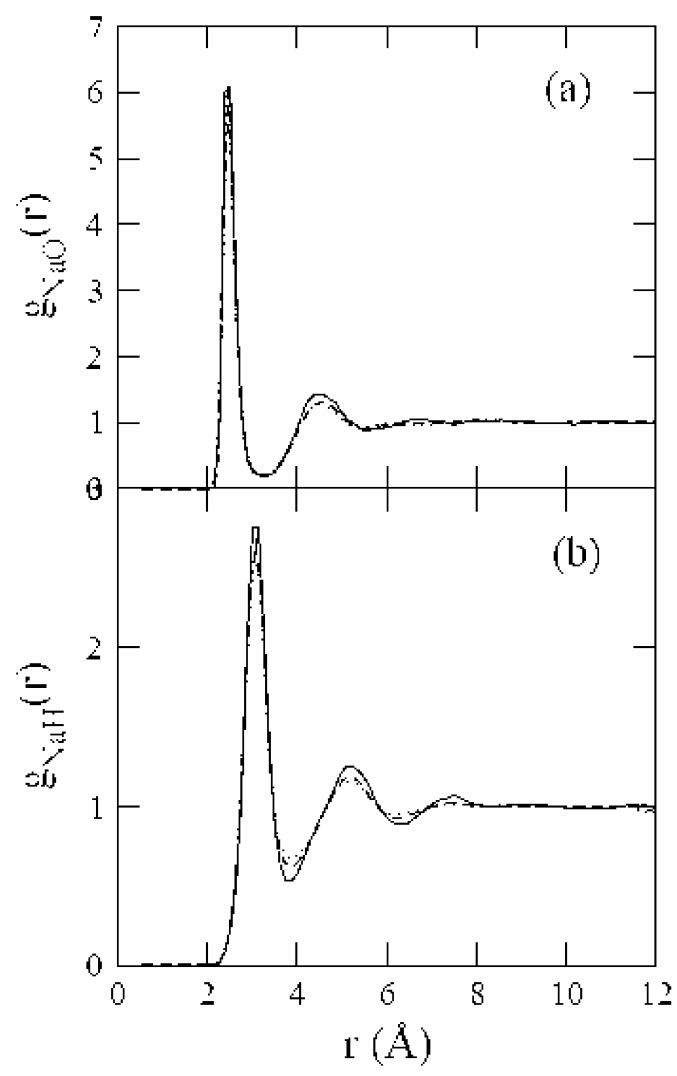

Figure 1. The sodium-oxygen and sodium-hydrogen radial distribution functions for $\mathrm{NaCl}$ solutions at $298 \mathrm{~K}$ for three different concentrations. The solid, dashed and dotted curves are for infinitely dilute, $3.486 \mathrm{M}$ and 5.326 M solutions respectively. 
second solvation shell radii. These values are determined from the locations of the first and second minima of the corresponding ion-oxygen radial distribution functions as shown in figures 1 and 2. It is seen from figure 1 that, for $\mathrm{Na}^{+}-\mathrm{O}$ pair correlations, although the peak heights change to some extent with increase of ion concentration, the locations of the first and second minima remain essentially unchanged and hence the shell radii were taken to be of the same value for all concentrations. The corresponding values for the $\mathrm{Cl}^{-}$were obtained from the $\mathrm{Cl}^{-}-\mathrm{O}$ radial distribution functions (figure 2). The radii for water solvation shells are, in a similar way, found from the water oxygen-oxygen correlations which are shown in figure 3 . We note that some changes are found in the locations of the first and second minima for both chloride ion-oxygen and

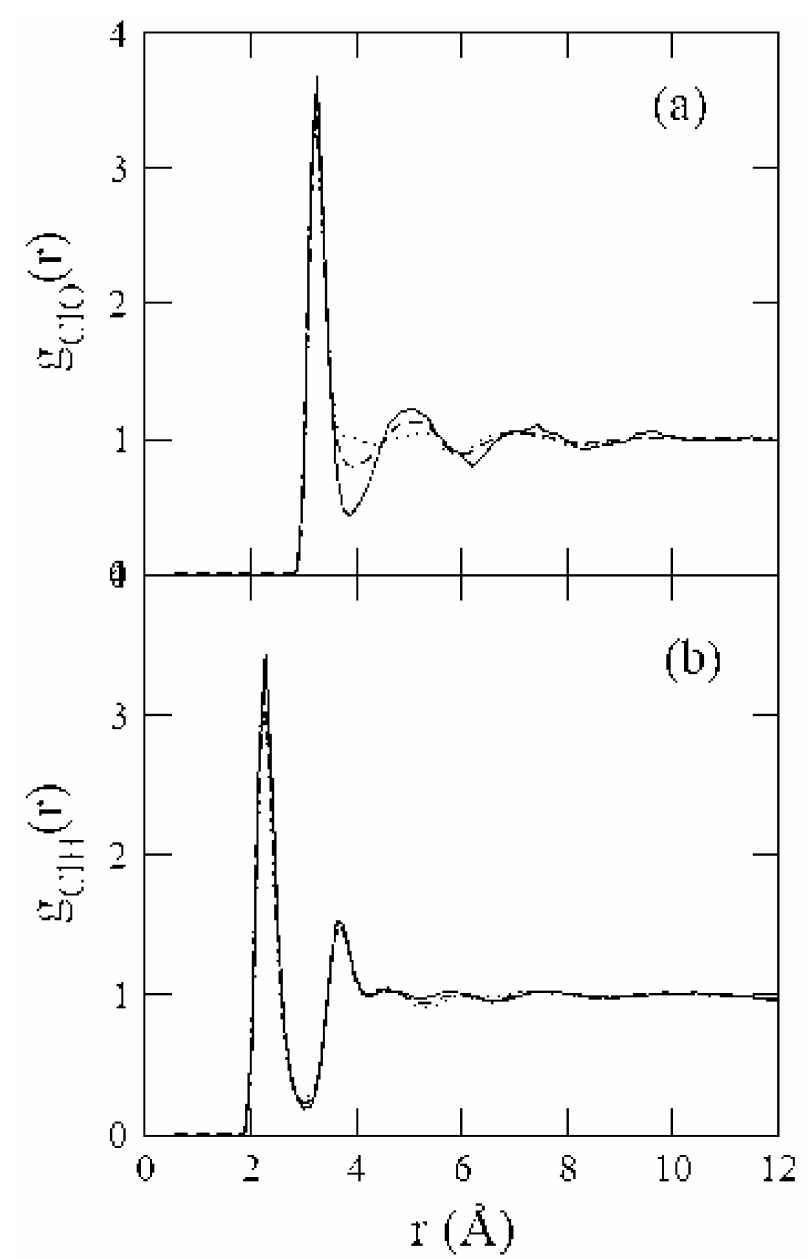

Figure 2. The chloride-oxygen and chloride-hydrogen radial distribution functions for $\mathrm{NaCl}$ solutions at $298 \mathrm{~K}$ for three different concentrations. The solid, dashed and dotted curves are for infinitely dilute, $3.486 \mathrm{M}$ and $5 \cdot 326 \mathrm{M}$ solutions respectively. oxygen-oxygen correlations with increase of ion concentration. However, to keep a common definition of the solvation shell boundaries, in all the cases the locations of the minima for the infinitely dilute solution were used.

In our analysis of hydrogen bonded structures, we employed a geometric definition of hydrogen bonds between two molecules or between an anion and a water. ${ }^{21-28}$ Two water molecules are said to be hydrogen bonded only if their interoxygen distance is less than $3.5 \AA$ and simultaneously hydrogenoxygen distance is less than $2.45 \AA$ and the oxygenoxygen-hydrogen angle is less than $45^{\circ}$. A chloride ion is said to be hydrogen bonded with a water molecule if the chloride-oxygen distance is less than $3.90 \AA$ and simultaneously the hydrogen-chloride distance is less than $3.05 \AA$ and the chloride-

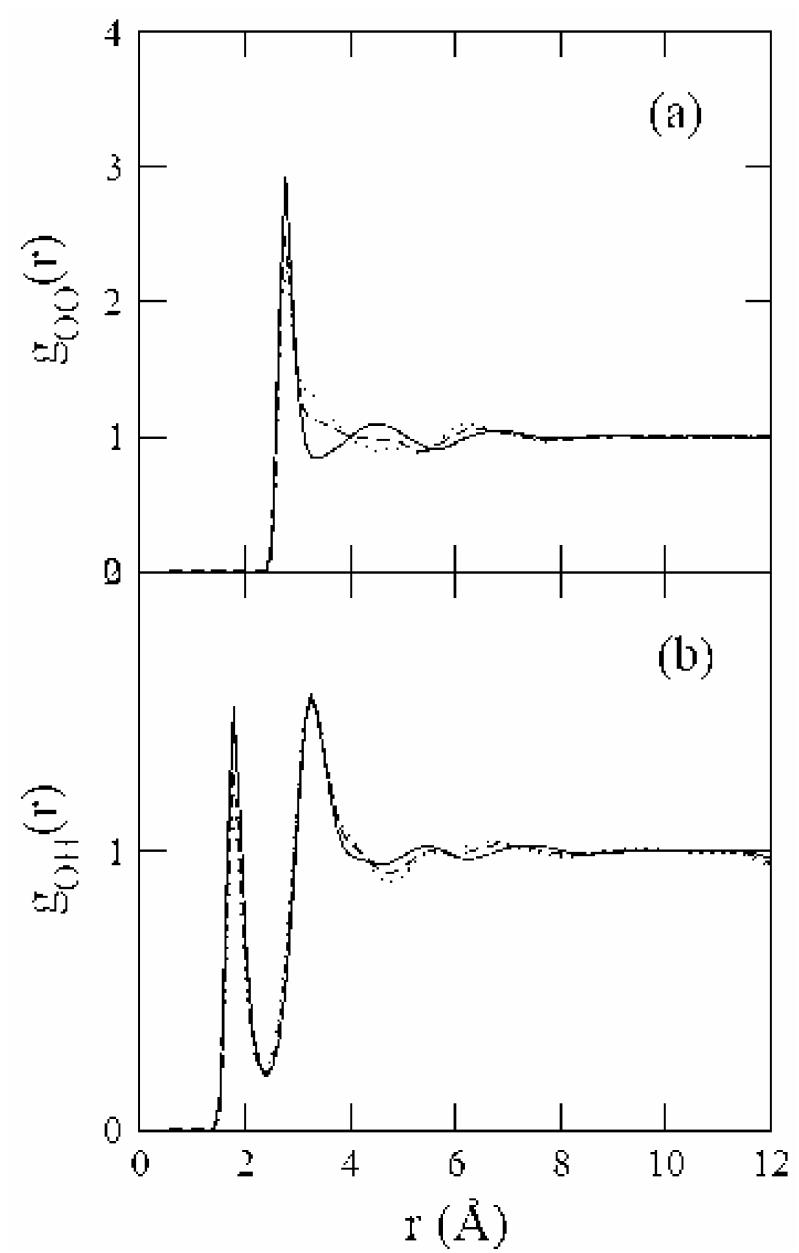

Figure 3. The oxygen-oxygen and oxygen-hydrogen radial distribution functions for $\mathrm{NaCl}$ solutions at $298 \mathrm{~K}$ for three different concentrations. The solid, dashed and dotted curves are for infinitely dilute, $3.486 \mathrm{M}$ and $5 \cdot 326 \mathrm{M}$ solutions respectively. 
oxygen-hydrogen angle is less than $45^{\circ}$. Although in ambient water, water-water and halide ion-water hydrogen bonds are found to be predominantly linear we have used an angular cut-off of $45^{\circ}$ to give allowance for fluctuations from the linear geometry which can occur due to thermal motion. The cut-off distances were determined from the relevant pair correlation functions of the infinitely dilute solutions. As discussed before, although some changes were found in radial distribution functions with increase of ion concentration, especially in oxygenoxygen and ion-oxygen correlations, we used the same cut-off values as for the dilute solutions in order to keep the definition of the hydrogen bonding the same. This would allow a meaningful compari-

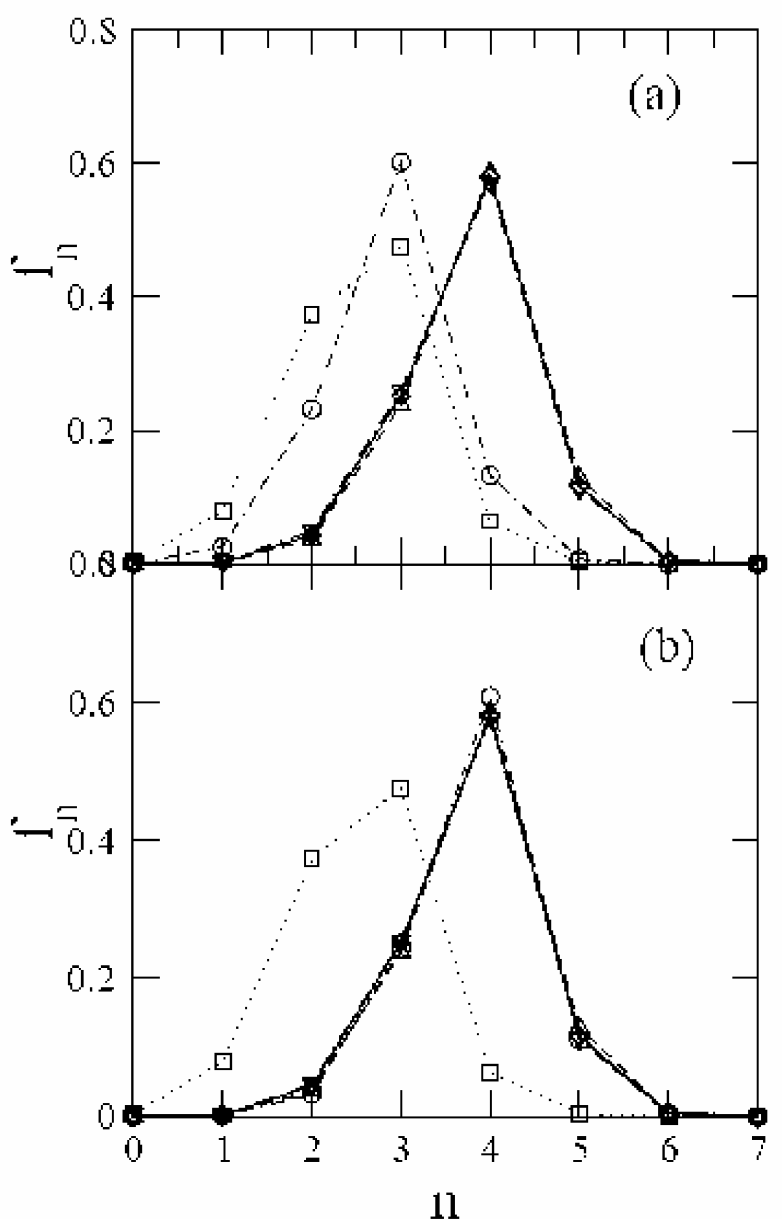

Figure 4. The fraction of water molecules having $n$ number of hydrogen bonds considering (a) water-water hydrogen bonds only and (b) both water-water and ionwater hydrogen bonds for infinitely dilute $\mathrm{NaCl}$ solution. The circles, squares, diamonds, triangle-up and triangledown are for $\mathrm{Cl}^{-}$first shell, $\mathrm{Na}^{+}$first shell, $\mathrm{Cl}^{-}$second shell, $\mathrm{Na}^{+}$second shell and bulk water, respectively. son of the hydrogen bond results for different ion concentrations.

For analysing the hydrogen bond network, we computed the fraction $\left(f_{n}\right)$ of water molecules that engage in $n$ water-water hydrogen bonds and the average number of hydrogen bonds $n_{H B}$ per water molecule considering only water-water hydrogen bonds in the first and second solvation shells of the ions. We also computed the fraction of water molecules that engage in a total of $n$ water-water and ion-water hydrogen bonds and the average number of hydrogen bonds per water molecule considering both ion-water and water-water hydrogen bonds in the first and second solvation shells of the ions. From a comparison of the two relevant sets of data,

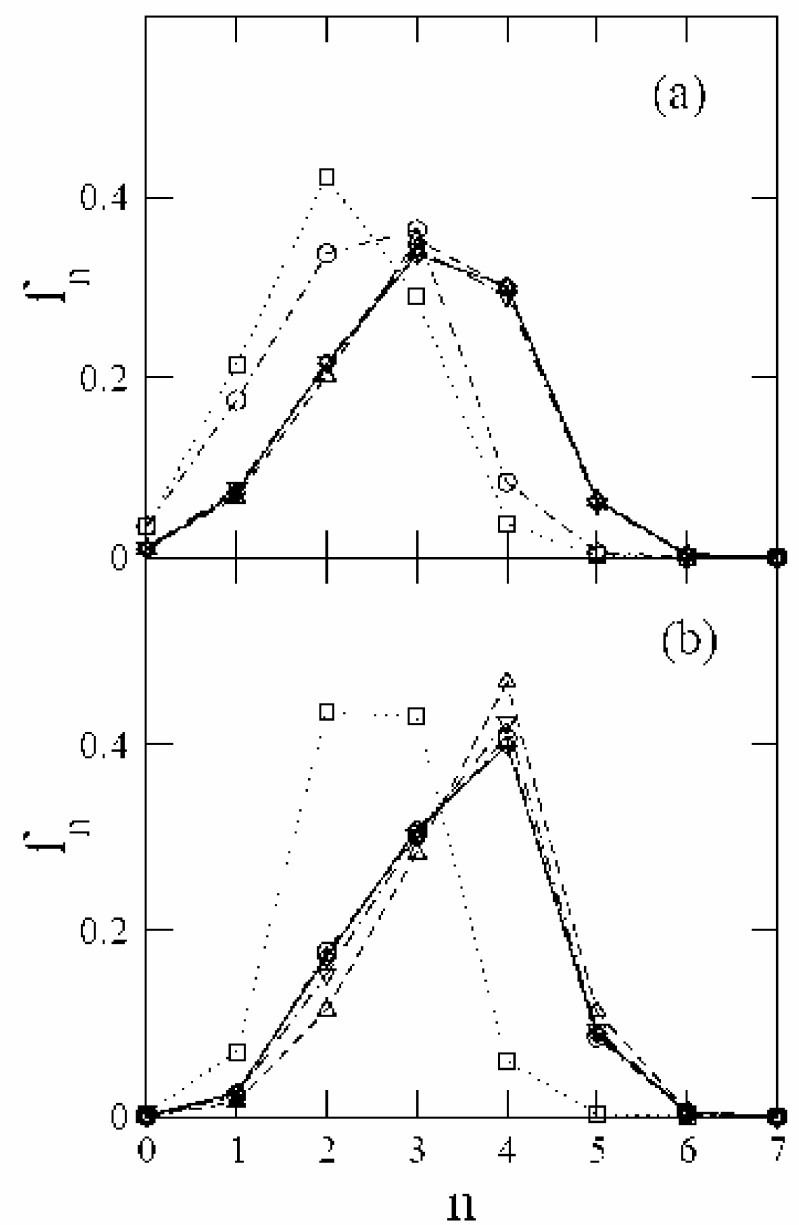

Figure 5. The fraction of water molecules having $n$ number of hydrogen bonds considering (a) water-water only and (b) both water-water and ion-water hydrogen bonds for $3.486 \mathrm{M} \mathrm{NaCl}$ solution. The circles, squares, diamonds, triangle-up and triangle-down are for $\mathrm{Cl}^{-}$first shell, $\mathrm{Na}^{+}$first shell, $\mathrm{Cl}^{-}$second shell, $\mathrm{Na}^{+}$second shell and bulk water, respectively. 
we could extract information about the ion-water hydrogen bonding contribution to the hydrogen bond connectivity. A similar calculation was done for the bulk water, i.e. averaged over all water molecules irrespective of whether they are inside or outside the solvation shells of the ions. From this we hope to address the question of whether there are any effects of ions on the hydrogen bond connectivity in the bulk water. The calculated distributions of $f_{n}$ for the first and second solvation shells of ions and also of bulk water are shown in figure 4 for the dilute solution. The corresponding results for the $3.5 \mathrm{M}$ and $5.3 \mathrm{M}$ solutions are presented in figures 5 and 6 , respectively. In each figure, the results are shown for (a) only water-water hydrogen bonds and also (b)

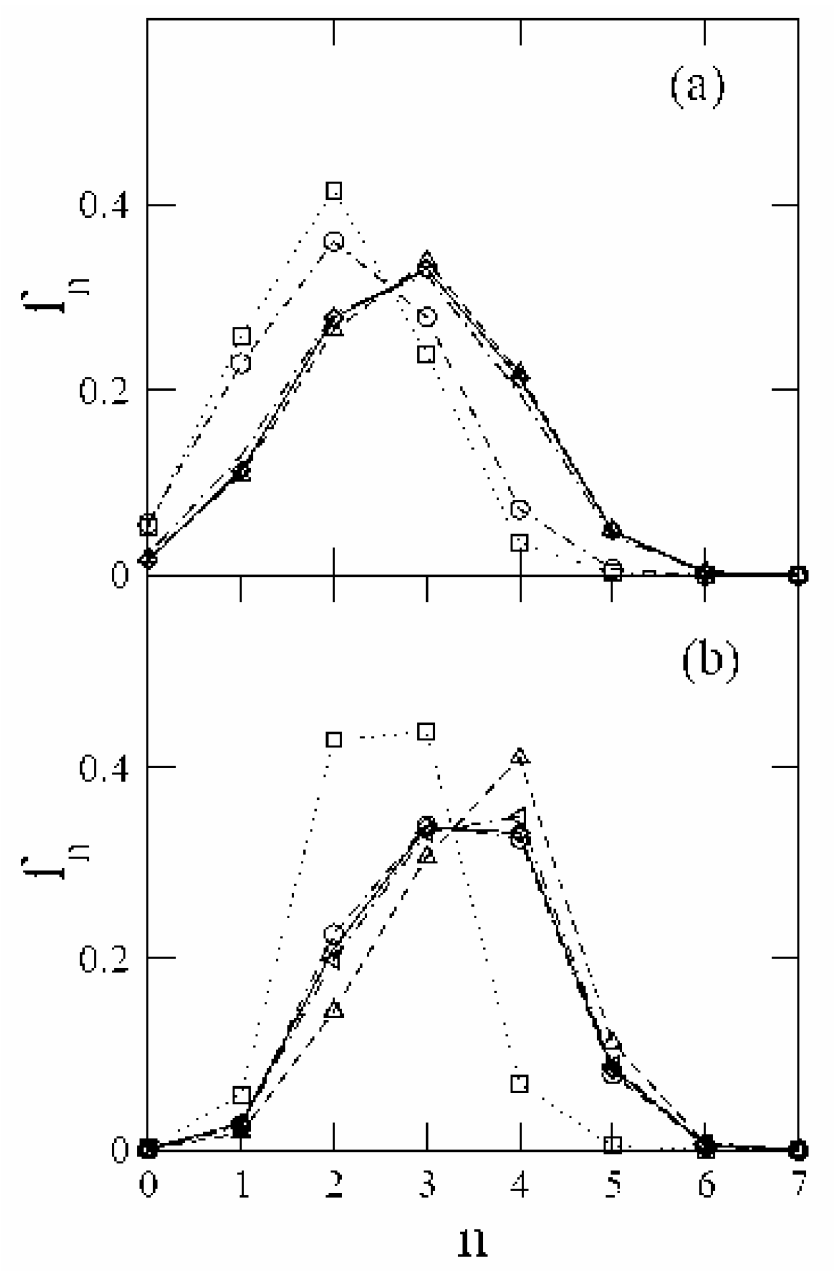

Figure 6. The fraction of water molecules having $n$ number of hydrogen bonds considering (a) water-water only and (b) both water-water and ion-water hydrogen bonds for $5.326 \mathrm{M} \mathrm{NaCl}$ solution. The circles, squares, diamonds, triangle-up and triangle-down are for $\mathrm{Cl}^{-}$first shell, $\mathrm{Na}^{+}$first shell, $\mathrm{Cl}^{-}$second shell, $\mathrm{Na}^{+}$second shell and bulk water, respectively. for the combination of water-water and anion-water hydrogen bonds. Our results for the average number of hydrogen bonds per water molecule in different solvation shells are summarized in table 2 .

It is found that the impact of ions on hydrogen bonding network is always more in the first solvation shells of the ions, $n_{\mathrm{HB}}$ being on an average, one less than that in the second solvation shells when we consider water-water hydrogen bonds only. This has been ascribed to the replacement of a water-water hydrogen bond by a hydrogen bond of type $\mathrm{X}^{-} \ldots \mathrm{H}-$ $\mathrm{O}$. Similar to the findings (ref. 14), we also find that the effects of the ionic species faded away in the second solvation shell for the infinitely dilute solution, considering the distributions $f_{n}$ and the average number of hydrogen bonds per water molecule. In fact, the contribution of ion-water hydrogen bonding was found to be significant only in the first solvation shells. However, for the concentrated solutions, we observed a significantly different scenario. Considering only water-water hydrogen bonding, the effect of ions on the hydrogen bond connectivity was found to be more significant in $3.486 \mathrm{M} \mathrm{NaCl}$ solution as compared to the infinitely dilute solution and was found to increase further for the $5.32 \mathrm{M}$ solution, with the average number of water-water hydrogen bonds per water molecule decreasing even in the bulk water. As expected, the extent of decrease was smaller for the second solvation shell and the bulk water as compared to the first solvation shell. Our calculations considering water-water as well as ion-water hydrogen bonding revealed that with increase in ionic concentration, ion-water hydrogen bonds replace water-water hydrogen bonds to some extent and the changes in the total number of hydrogen bonds per water molecule in concentrated solutions are not as great as was found when only the water-water hydrogen bonds were considered. We have also looked at the hydrogen bond statistics of water molecules in the solvation shells of the cation. It is found that, in the infinitely dilute solution, there were no contribution of anion-water hydrogen bonding to $n_{\mathrm{HB}}$ in the first and second solvation shells of the cation. But for the concentrated solutions, a distinct contribution of the former is found to be indicating the presence of solvent separated ion-pairs at high concentrations.

A comparison of the distribution of the fraction of water molecules that engage in hydrogen bonds for the two solvation shells and bulk water for a particular concentration also revealed certain trends. For infinitely dilute $\mathrm{NaCl}$ solution, the distribution was 
Table 2. The average number of hydrogen bonds per water molecule $n_{\mathrm{HB}}$ in infinitely dilute, $3.486 \mathrm{M}$ and $5.326 \mathrm{M}$ $\mathrm{NaCl}$ solutions at $298 \mathrm{~K}$.

\begin{tabular}{lccccc}
\hline & $\mathrm{Cl}^{-}$first shell & $\mathrm{Cl}^{-}$second shell & $\mathrm{Na}^{+}$first shell & $\mathrm{Na}^{+}$second shell & Bulk water \\
\hline Water-water infinitely dilute & 2.86 & 3.78 & 2.53 & 3.82 & 3.75 \\
$3.486 \mathrm{M}$ & 2.30 & 3.05 & 2.09 & 3.07 & 3.02 \\
$5.326 \mathrm{M}$ & 2.10 & 2.76 & 1.96 & 2.79 & 2.68 \\
Water-water and ion-water & & & & & \\
infinitely dilute & 3.81 & 3.78 & 2.53 & 3.82 & 3.76 \\
$3.486 \mathrm{M}$ & 3.36 & 3.36 & 2.48 & 3.56 & 3.42 \\
$5.326 \mathrm{M}$ & 3.22 & 3.25 & 2.53 & 3.47 & 3.29 \\
\hline
\end{tabular}

found to be nearly identical for the second solvation shells of the ions and the bulk water. When we considered the water-water and ion-water hydrogen bonding, excepting the first solvation shell of the cation, which remained unchanged, the distributions became essentially identical, indicating the presence of ion-water hydrogen bonding in the first solvation shell of the anion and absence of ion-water hydrogen bonding in the first solvation shell of the cation. For the concentrated $\mathrm{NaCl}$ solutions, the distribution of the first solvation shell of the cation was found to be markedly different from the other distributions. Certain contributions of the ion-water hydrogen bonding in the first solvation shell of the cation were found to be present in these cases. For the other distributions in concentrated $\mathrm{NaCl}$ solutions, on considering the water-water as well as ion-water hydrogen bonding, the distributions tended to converge with each other but the extent of overlap of the distributions were less for high concentrations compared to that of the infinitely dilute solution. The extent of variation increased with an increase in ion concentration. This also supports our view that, although at infinite dilution ions do not appear to affect the hydrogen bonding network beyond the first solvation, such effects at high concentrations are not fully negligible.

\section{Conclusions}

In this study we have presented molecular dynamics simulations of aqueous $\mathrm{NaCl}$ solutions of varying concentrations under ambient conditions. The aim of this study has been to address the issue of how ions alter the hydrogen bonded network of water at different concentrations. To this end we have analysed the equilibrium distributions of hydrogen bonds in the first and second solvation shells of the ions as well as in bulk water and also computed the average number of hydrogen bonds per water molecule, once considering only water-water hydrogen bonds and in the other case considering both water-water as well as ion-water hydrogen bonds.

Our results reveal that unlike for the infinitely dilute solutions, in the concentrated solutions there is a non-negligible effect of ions on the hydrogen bonding network beyond the first solvation shells of the ions, i.e. in the second salvation shells. Even in the bulk water there is an influence, though small, of the ions on the hydrogen bonding network. This effect is more prominent if we consider water-water hydrogen bonding only. With increase in concentration, ionwater hydrogen bonds are formed in place of some of the water-water hydrogen bonds. But even if we consider both ion-water and water-water hydrogen bonds, we can see an effect of ions on the hydrogen bonding of water which is not fully negligible. Although our results are in general accordance with recent studies regarding the not-so-important effects of ions on the hydrogen bonded structure beyond the first solvation shell, the present study reveals that water-water network can be affected for highly concentrated solutions and the combined statistics of water-water and anion-water hydrogen bonds changes relatively weak with ion concentration compared to that of only water-water hydrogen bonds.

\section{Acknowledgement}

We gratefully acknowledge financial supports from the Department of Science and Technology (DST), Department of Atomic Energy (DAE) and Council of Scientific and Industrial Research (CSIR), Government of India. We thank B S Mallik for help in the initial stage of the simulations. 


\section{References}

1. Mesmer R E, Sweeton F H, Hitch B F and Baes C F 1976 In High-temperature high-pressure electrochemistry in aqueous solutions (eds) D D G Jones and R W Staehle (Houston, TX: National Association of Corrosion Engineers) pp 365-374

2. Marshall W L and Frantz J D 1980 In Thermodynamics of aqueous systems with industrial applications (ed.) S A Newman (Washington DC: American Chemical Society); vol 133, pp 569-581

3. Walfaren G E 1966 J. Chem. Phys. 441546

4. Walfaren G E 1970 J. Chem. Phys. 524176

5. Amo Y and Tominaga Y 2000 Physica A275 33

6. Smith J D, Saykally R J and Geissler P $2007 \mathrm{~J}$. Am. Chem. Soc. 12913847

7. Pfund D M, Darab J G, Fulton J L and Ma Y $1994 J$. Phys. Chem. 9813102

8. Wallen S L, Pfund D M and Fulton J L 1998 J. Chem. Phys. 1084039

9. de Jong $\mathrm{P} \mathrm{H} \mathrm{K}$, Neilson $\mathrm{G} \mathrm{W}$ and Bellissent-Funel M C 1996 J. Chem. Phys. 1055155

10. Yamaguchi T, Yamagami M, Ohzono $\mathrm{H}$, Wakita $\mathrm{H}$ and Yamanaka K 1996 Chem. Phys. Lett. 252317

11. Mancinelli R, Botti A. Bruni F, Ricci M A and Soper A K 2007 Phys. Chem. Chem. Phys. 92959

12. Omta A W, Kropman M F, Wouterson S and Bakker H J 2003 Science 301347

13. Omta A W, Kropman M F, Wouterson S and Bakker H J 2003 J. Chem. Phys. 11912457
14. Guardia E, Laria D and Marti J 2006 J. Phys. Chem. B110 6332

15. Du H, Rasaiah J C and Miller J D 2007 J. Phys. Chem. 111209

16. Berendsen H J C, Grigera J R and Straatsma T P 1987 J. Phys. Chem. 916269

17. Koneshan S, Rasaiah G C, Lyndel-Bell R M and Lee S H 1998 J. Phys. Chem. B102 4193

18. Smith D E and Dang L X 1994 J. Chem. Phys. 100 3757; Dang L X 1995 J. Chem. Phys. 1023483

19. Weast R C et al (eds) 1989 CRC handbook of chemistry and physics (Florida: CRS Press, Boca Raton)

20. Allen M P and Tildesley D J 1987 Computer simulation of liquids (Oxford)

21. Chandra A 2003 J. Phys. Chem. 1073899

22. Chowdhury S and Chandra A 2006 J. Phys. Chem. B31 159

23. Sutmann G and Vallauri R 1998 J. Phys: Condensed Matter 109231

24. Luzar A and Chandler D 1996 Phys. Rev. Lett. 76 928; Luzar A and Chandler D 1996 Nature (London) 37953

25. Xu H and Berne B J 2001 J. Phys. Chem. B105 11929; Xu H, Stern H A and Berne B J 2002 J. Phys. Chem. B106 2054; Luzar A 2000 J. Chem. Phys. 113 10663

26. Chandra A 2000 Phys. Rev. Lett. 85768

27. Balasubramanian S, Pal S and Bagchi B 2002 Phys. Rev. Lett. 89115505

28. Luzar A 2000 J. Chem. Phys. 11310663 